\title{
Weaver syndrome
}

INSERM

\section{Source}

INSERM. (1999). Orphanet: an online rare disease and orphan drug data base. Weaver syndrome. ORPHA:3447

Weaver syndrome (WVS) is a rare, multisystem disorder characterized by tall stature, a typical facial appearance (hypertelorism, retrog nathia) and variable intellectual disability. Additional features may include camptodactyly, soft doughy skin, umbilical hernia, and a low hoarse cry. 